BMJ Open Diabetes

Research \& Care

\title{
Exploring geographic variation of and influencing factors for utilization of four diabetes management measures in Swiss population using claims data
}

\author{
Wenjia Wei (1) , ${ }^{1}$ Oliver Gruebner, ${ }^{1,2}$ Viktor von Wyl, ${ }^{1}$ Holger Dressel, ${ }^{3}$ Agne Ulyte,${ }^{1}$ \\ Beat Brüngger (D) , ${ }^{1,4}$ Eva Blozik, ${ }^{4,5}$ Caroline Bähler, ${ }^{1,4}$ Julia Braun, ${ }^{6}$
} Matthias Schwenkglenks ${ }^{1}$

To cite: Wei W, Gruebner 0 , von Wyl V, et al. Exploring geographic variation of and influencing factors for utilization of four diabetes management measures in Swiss population using claims data. BMJ Open Diab Res Care 2020;8:e01059. doi:10.1136/ bmjdrc-2019-001059

- Additional material is published online only. To view please visit the journal online (http://dx.doi.org/10.1136/ bmjdrc-2019-001059).

Received 18 November 2019 Revised 31 December 2019 Accepted 22 January 2020
Check for updates

(C) Author(s) (or their employer(s)) 2020. Re-use permitted under CC BY-NC. No commercial re-use. See rights and permissions. Published by BMJ.

For numbered affiliations see end of article.

Correspondence to

WenjiaWei; wenjia.wei@uzh.ch

\section{ABSTRACT}

Introduction Four strongly recommended diabetes management measures are biannual glycated hemoglobin (HbA1c) testing, annual eye examination, kidney function examination, and low-density lipoprotein (LDL) testing in patients below 75 years. We aimed to describe regional variation in the utilization of the four measures across small regions in Switzerland and to explore potential influencing factors.

Research design and methods We conducted a cross-sectional study of adult patients with drugtreated diabetes in 2014 using claims data. Four binary outcomes represented adherence to the recommendations. Possible influencing factors included sociodemographics, health insurance preferences, and clinical characteristics. We performed multilevel modeling with Medstat regions as the higher level. We calculated the median odds ratio (MOR) and checked spatial autocorrelation in region level residuals using Moran's I statistic. When significant, we further conducted spatial multilevel modeling.

Results Of 49198 patients with diabetes (33957 below 75 years), $69.6 \%$ had biannual $\mathrm{HbA} 1 \mathrm{c}$ testing, $44.3 \%$ each had annual eye examination and kidney function examination, and $55.5 \%$ of the patients below 75 years had annual LDL testing. The effects of health insurance preferences were substantial and consistent. Having any supplementary insurance (ORs across measures were between 1.08 and 1.28), having supplementary hospital care insurance (1.08-1.30), having chosen a lower deductible level (eg, SFr2500 compared with SFr300: 0.57-0.69), and having chosen a managed care model (1.04-1.17) were positively associated with recommendations adherence. The MORs (1.27-1.33) showed only moderate unexplained variation, and we observed inconsistent spatial patterns of unexplained variation across the four measures.

Conclusion Our findings indicate that the uptake of strongly recommended measures in diabetes management could possibly be optimized by providing further incentives to patients and care providers through insurance scheme design. The absence of marked regional variation implies limited potential for improvement by targeted regional intervention, while provider-specific promotion may be more impactful.

\section{Significance of this study}

What is already known about this subject?

- Better adherence to clinical guidelines and recommendations could increase clinical outcomes of patients with diabetes.

- Little is known about the utilization and its regional variation of recommended measures in diabetes management.

What are the new findings?

- In a healthcare system with mandatory health insurance, choosing a lower deductible level, choosing a managed care model, and having supplementary insurance were associated with better adherence.

- Unexplained variation after adjusting for possible influencing factors was not pronounced, and inconsistent spatial patterns were observed across different measures.

How might these results change the focus of research or clinical practice?

- The findings imply a potential to optimize the utilization of recommended healthcare services by providing further incentives through insurance scheme design.

\section{INTRODUCTION}

Diabetes is one of the most common chronic diseases. The global prevalence in adults over 18 years was $8.5 \%$ (around 422 million) in 2014 , and deaths directly caused by diabetes were estimated at 1.6 million in $2016 .^{1}$ In Switzerland, an estimated 500000 persons suffer from the condition, which is responsible for around $2 \%$ of all deaths. ${ }^{2}$ Diabetes can be treated and its complications delayed through various measures, including constant medical care, restricted diet, physical activity, and regular screening. ${ }^{3}$

A variety of clinical guidelines on diabetes management have been developed nationally 
and internationally to improve outcomes. The Swiss Society of Endocrinology and Diabetology (SGED) has developed "The criteria for good disease management of Diabetes in primary care" in 2013 and revised it in 2017. ${ }^{4}$ The American Diabetes Association, the European Society of Cardiology, and the European Association for the Study of Diabetes report and annually adapt clinical guidelines on diabetes as well. ${ }^{56}$ Some recommendations on diabetes management are crucial and consistently present in almost all clinical guidelines, for instance, biannual glycated hemoglobin (HbAlc) testing, annual eye examination, and low-density lipoprotein (LDL) testing. Studies have shown strong evidence that the complications of diabetes could be reduced through managing risk factors such as increased HbA1c, LDLs, and blood pressure. ${ }^{7-9}$ It has also been reported that adherence to clinical guidelines had a positive influence on clinical outcomes including mortality and hospitalizations. ${ }^{10-12}$

In the present study, we used four strong standard recommendations included in most diabetes clinical guidelines, that is, patients with diabetes should undergo (1) at least two HbA1c tests per year, (2) at least one eye examination per year, (3) at least one kidney function examination per year, and (4) at least one LDL test per year (only in patients below 75 years). One study in Switzerland using health insurance claims data for the years 2011-2013 reported overall adherence to these recommendations but did not perform an in-depth assessment of drivers of utilization or geographic variation. ${ }^{13}$ Generally, few studies have investigated the adherence to clinical recommendations on diabetes management.

Various factors may affect the utilization of healthcare services for diabetes, including characteristics of patients, healthcare providers, health insurance, and regions. This could result in geographic variation in utilization. Such variation in utilization may be unwarranted, which would to some extent reflect unequal access. ${ }^{14}$ The four diabetes recommendations are clear and based on highquality evidence, and the corresponding management measures are not preference sensitive. ${ }^{15}$ Moreover, access to healthcare in general and the four measures in particular is very good in Switzerland. Therefore, we expected little unexplained variation after adjusting for possible influencing factors in the present case. ${ }^{16}$

The aims of this study were to (1) investigate the utilization levels of the above-defined strongly recommended measure in diabetes management, (2) explore potential factors influencing utilization, and (3) assess the regional variation in utilization of the four measures across small regions in Switzerland.

\section{MATERIALS AND METHODS}

\section{Study population}

We used health insurance claims data provided by the Helsana Group, one of the largest health insurance companies in Switzerland. The Helsana database underlying this study included mandatory health insurance claims from around 1.2 million people, covering 15\% of the Swiss population. Adults (older than 18 years) enrolled with Helsana who were prescribed any diabetes medication (the Anatomical Therapeutic Chemical code was used to identify diabetes medications) between January 1, 2014 and December 27, 2014 were analyzed. We excluded enrollees with incomplete insurance coverage in 2014 or not surviving until the end of 2014, patients living outside Switzerland, asylum seekers, Helsana employees, patients with incomplete address information, and patients living in nursing homes with lump-sum reimbursement. Since diagnosis information was not available for outpatient services, we could not distinguish between type 1 and type 2 diabetes.

Basic health insurance (covering a federally defined benefit package) is mandatory in Switzerland and private insurance companies are obliged to offer it to anyone irrespective of their health status. Mandatory health insurance includes appropriate and cost-effective inpatient and outpatient health services. A variety of annual deductibles (300-2500 Swiss Francs (SFr)) can be chosen, and selecting a higher deductible leads to a lower premium. Enrollees can also choose between standard and managed care models, where the latter require a specific general practitioner or telemedicine provider as the first contact when a new health problem arises, hence are cheaper. ${ }^{17}{ }^{18}$ In addition to mandatory health insurance, a variety of supplementary health insurance products can be bought, for instance, supplementary hospital care insurance which allows for hospitalization in semiprivate/private wards. ${ }^{17}$

\section{Outcome and explanatory variables}

We differentiated the participants according to whether they received diabetes medication between January 5 , 2013 and December 31, 2013 (prevalent cases if they received it, incident cases if they did not). The date of the first prescription of any diabetes medication in 2014 (incident cases) or January 1, 2014 (prevalent cases) was considered as the index date for each participant. The following 360 days were regarded as the assessment period, which was used to define if the recommendations were being adhered to.

We defined binary outcome variables for the four measures under study: in the assessment period, (1) at least two HbA1c tests, (2) at least one eye examination (ophthalmologist visit was used as a proxy of eye examination), (3) at least one kidney function examination (ie, serum creatinine and/or albuminuria test), and (4) at least one LDL test (or total cholesterol+high-density lipoprotein (HDL)+triglyceridestest) for patients below 75 years. ${ }^{19}$

The explanatory variables included (1) sociodemographics, including age, gender, language region, purchasing power index per household, and urban/rural residence; (2) health insurance preferences, including having both mandatory and supplementary insurance, having supplementary hospital care insurance, standard 
or managed care model, and choice of annual deductible; and (3) clinical characteristics, including number of comorbidities (pharmaceutical cost groups were used to deduce chronic morbidity based on drug use) ${ }^{20}$ and incident or prevalent diabetes treated with oral medication or insulin. In addition, a region-level variable-ophthalmologist density per 10000 inhabitants—was used specifically for the study of eye examinations.

\section{Geographic unit}

We used Medstat regions as the geographic units for regional variation analysis. Medstat regions $(\mathrm{n}=705)$ are defined by the Swiss Federal Statistical Office to reflect hospital catchment areas in a way that they are large enough to provide anonymity for each person hospitalized in Switzerland. ${ }^{21}$ Each patient's residence was assigned to the corresponding Medstat region in the claims data.

\section{Statistical analysis}

First, we performed a descriptive analysis of study population's characteristics. We distinguished between all eligible patients and the subpopulation below 75 years, relevant for the assessment of LDL testing.

Second, we mapped out the raw utilization rates of the four measures across Medstat regions to show their geographic distribution. We checked spatial autocorrelation of regional utilization rates by computing the global Moran's I statistic. ${ }^{22}$ Moran's I measures the correlation of a variable with itself through space, with a value range from -1 to 1 . Moran's I values very close to 0 suggest the studied variable is randomly distributed through space. If Moran's I is positive with $\mathrm{p}$ value $<0.05$, it indicates that neighboring regions are more similar than distant regions, and vice versa if Moran's $I$ is negative with $p$ value $<0.05$.

Third, we performed multilevel multivariable logistic regression for each measure, with patients as the lowerlevel units and Medstat regions as the higher-level units. Decisions on inclusion of explanatory variables were based on the deviance information criterion. ${ }^{23}$ We included age as a quadratic term to allow for nonlinear relationships. The Medstat region-level variable of ophthalmologist density per 10000 inhabitants was included in the model for eye examination. To estimate the degree of random variation in the multilevel models, we calculated the median odds ratio (MOR) at the Medstat region level. The MOR compares the adjusted odds of being adherent to the recommendation in two patients with identical characteristics, but living in two randomly selected Medstat regions. It is defined as the median of all possible, resulting ORs. The MOR is never below one as the comparison is always between the higher-propensity region and the lower-propensity region, for the outcome of interest. ${ }^{24-26} \mathrm{~A}$ higher MOR indicates a higher level of unexplained variation in utilization after multivariable adjustment, and it can be compared directly with the ORs of the fixed effects. ${ }^{24-26}$
We then checked for the presence of spatial autocorrelation in the model residuals at the Medstat region level, for each measure. ${ }^{22}$

Finally, in cases with significant spatial autocorrelation present in the multilevel model residuals, we further developed Bayesian hierarchical logistic regression models capturing spatial variation at the Medstat region level through the Integrated Nested Laplace Approximations (INLA) approach. ${ }^{27-30}$ This was performed with the R-INLA package. ${ }^{31}$ The covariates included in the spatial multilevel models were the same as in the multilevel multivariable models above. The marginal effects of age divided into 50 groups were inspected graphically, which was more intuitive than reporting regression coefficients. The finally remaining geographic variation across Medstat regions was mapped out for each measure.

Statistical analyses were performed using R V.3.4.4, ${ }^{32}$ STATA V.13, and MLwiN V.3.04 ${ }^{33}$ integrated in STATA using the runmlwin package. Mapping was conducted with QGIS V.1.14.16, ${ }^{34}$ and spatial clustering analysis was done with GeoDa V.1.10. ${ }^{35}$

\section{RESULTS}

A total of 49198 patients with diabetes were analyzed in this study. The mean age was 66.6 years, and women accounted for $45.0 \%$ of the whole study sample. Overall, $34254(69.6 \%)$ patients had at least two HbAlc tests in their assessment period, 21808 (44.3\%) patients had at least one kidney function examination, and 21804 (44.3\%) patients had at least one eye examination. Among the 49198 patients with diabetes, 33957 were below 75 years and were analyzed for LDL testing. In this subpopulation, $18851(55.5 \%)$ patients had at least one LDL test in the assessment period. The mean age was 60.1 years, and $41.2 \%$ were women. Table 1 shows the sociodemographics, health insurance preferences and clinical characteristics of the total population and of those below 75 years, respectively.

The ORs and $95 \%$ confidence/credible intervals $(95 \%$ CIs) of all explanatory variables (except age, shown in figure 1) in both the multilevel multivariable models and the spatial multilevel models are shown in figure 2 (full numerical details are available from online supplementary table S1 and S2). For each pair of models representing one outcome, covariate effects were similar, except for language region. Regarding sociodemographics, women were more likely to follow the recommendations of eye examination and kidney function examination, while the opposite was true for LDL testing. Purchasing power index was positively associated with eye examination and kidney function examination, while it was negatively associated with HbAlc testing. Living in an urban area had a positive association with kidney function examination and LDL testing. Compared with the German-speaking area, living in the French or Italian speaking area of Switzerland demonstrated a negative association with HbAlc testing and eye examination, as well as positive 


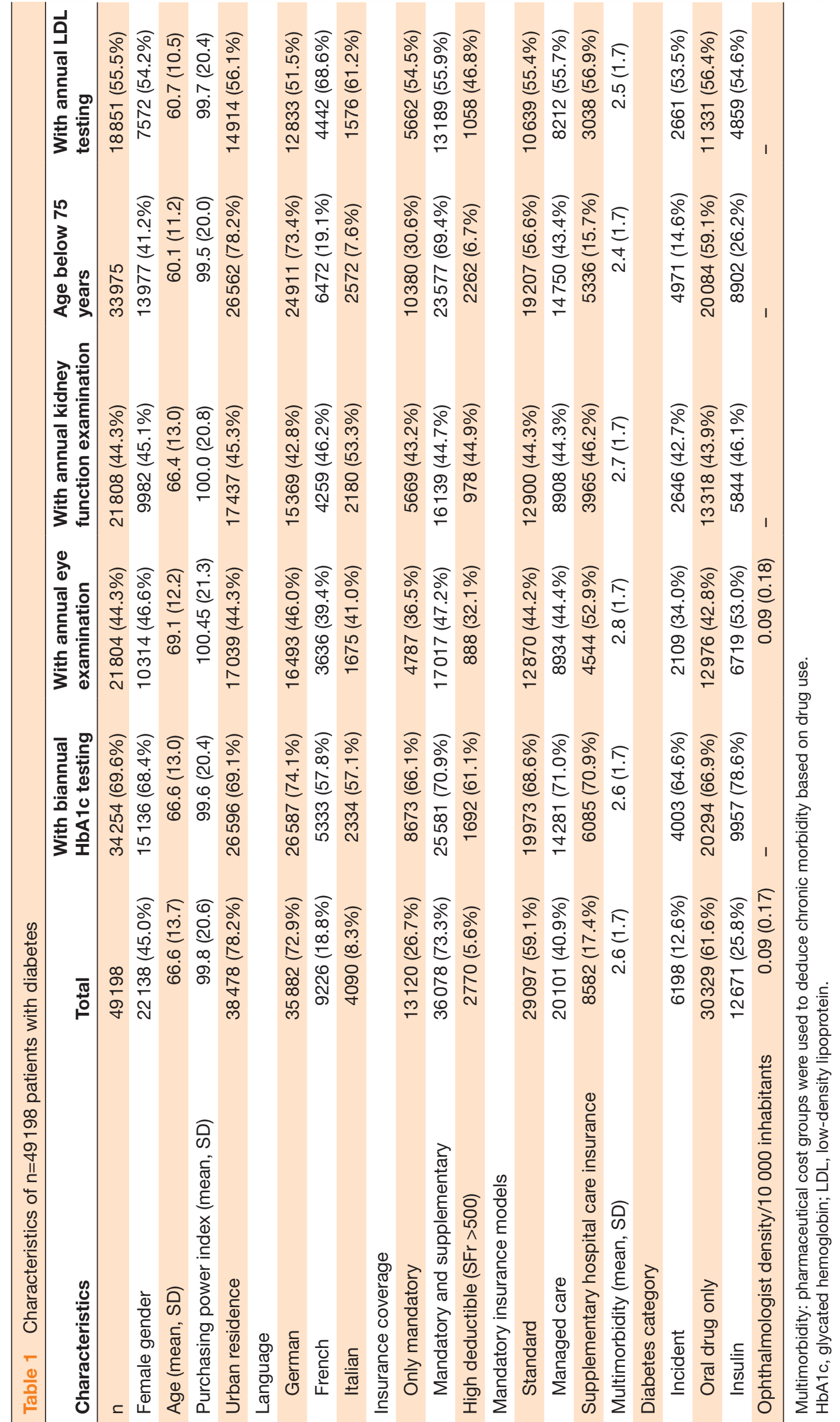


HbA1c testing

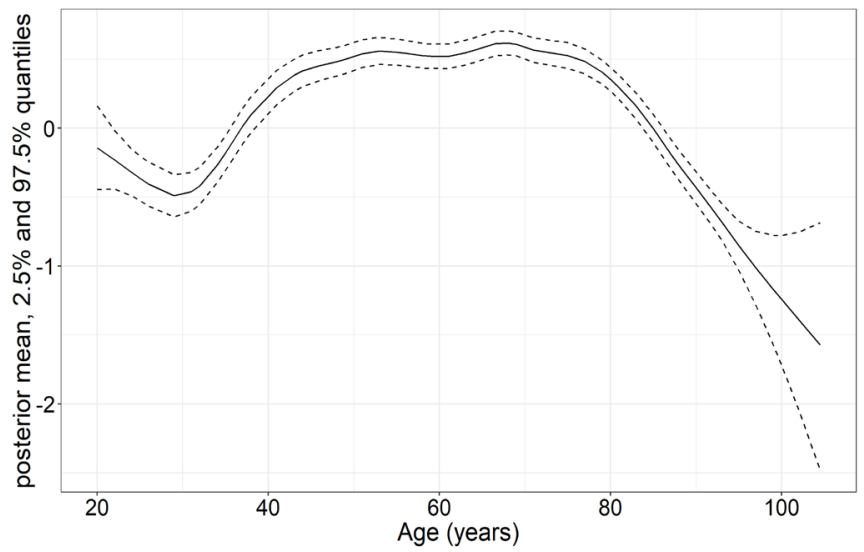

Kidney function examination

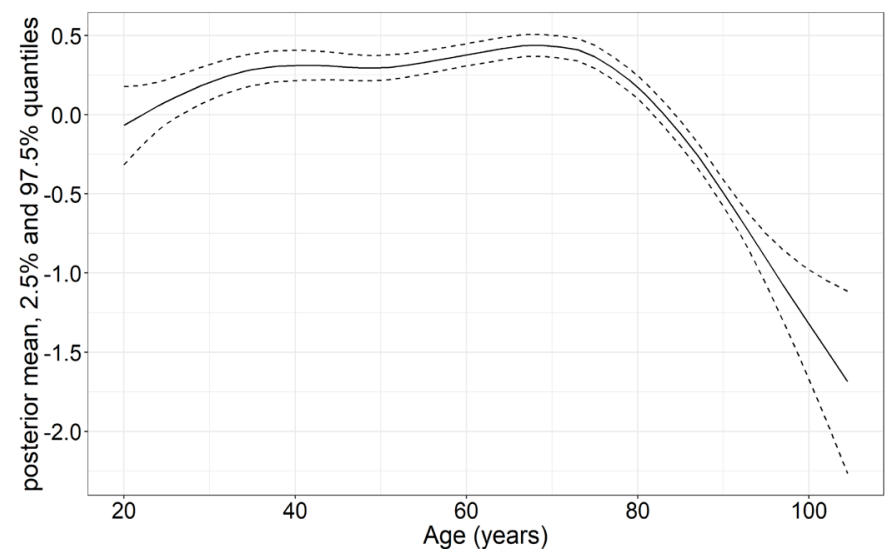

Eye examination

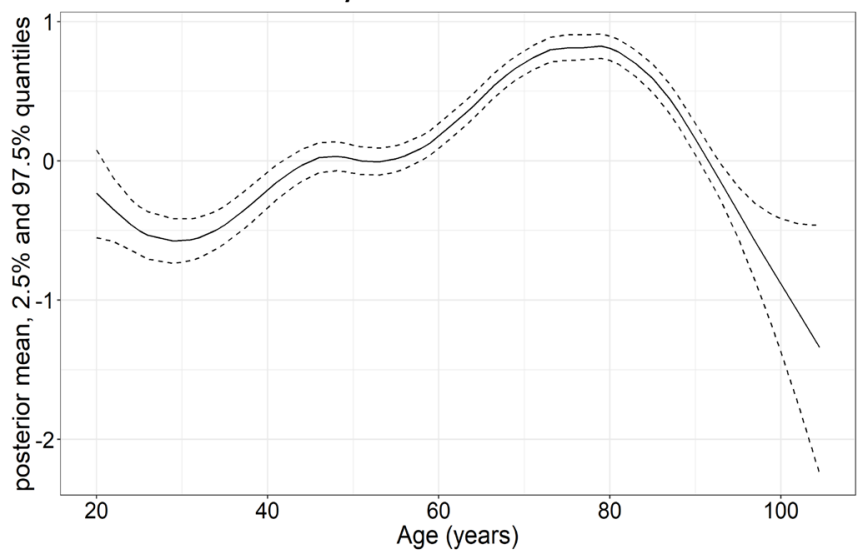

LDL testing

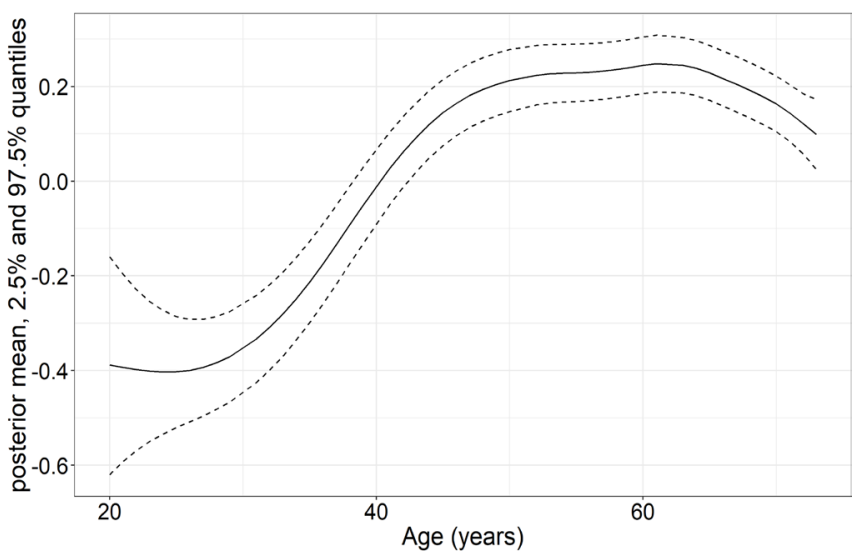

Figure 1 Age effect in spatial multilevel models. HbA1c, glycated hemoglobin; LDL, low-density lipoprotein.

associations with LDL testing and kidney function examination. As expected, these associations were strongly attenuated in the spatial multilevel models. The effects of health insurance preferences and clinical characteristics were mostly consistent across the four measures. Having any supplementary insurance (ORs across measures were between 1.08 and 1.28), having supplementary hospital care insurance (1.08-1.30), having chosen a managed care insurance model (1.04-1.17), and having more comorbidities (eg, having more than two morbidities compared with none: $1.25-1.57$ ), were all positively associated with being adherent to the recommendations. Having chosen a higher deductible level had a negative association with being adherent to the recommendations (eg, SFr2500 compared with SFr300: 0.57-0.69). Prevalent cases receiving insulin compared with incident cases had a positive association with HbA1c testing, eye examination and kidney function examination, while having a negative association with LDL testing. A positive association between ophthalmologist density and eye examination was found in both models of eye examinations, with an OR of 1.13 in the spatial multilevel model.

The geographic distribution of the utilization of the four measures across Medstat regions is shown in figure 3 . By visually inspecting the maps, we noted considerable geographic variation for each measure, with quite different spatial patterns. For HbA1c testing, utilization rates were generally higher in the German-speaking north and middle part of Switzerland, while there was no such pattern visible for the other three measures. Positive and statistically significant $(\mathrm{p}<0.05)$ Moran's I values of the raw rates indicating the presence of spatial autocorrelation were 0.46 for $\mathrm{HbAlc}$ testing, 0.27 for eye examination, 0.21 for kidney function examination, and 0.35 for LDL testing. After multilevel multivariable model adjustment, Moran's I values of the residuals at the Medstat region level decreased to $0.12(\mathrm{p}<0.0001)$ for HbA1c testing, 0.13 ( $\mathrm{p}=0.0001)$ for kidney function examination, and $0.07(\mathrm{p}=0.004)$ for LDL testing. Only for eye examination, we found a slight increase of spatial autocorrelation to $0.30(\mathrm{p}<0.001)$ after model adjustments. In consequence, we regarded the spatial multilevel models as most appropriate because they accounted for the spatial structure in the data. The MORs in the multilevel multivariable models, describing variation between Medstat regions, were 1.28 for $\mathrm{HbAlc}$ testing, 1.31 for eye examination, 1.27 for kidney function examination, and 1.33 for LDL testing.

The unexplained spatial variation remaining after multivariable adjustment through spatial multilevel models for each measure is shown in figure 4. The OR values in figure 4 represent the odds of being adherent 


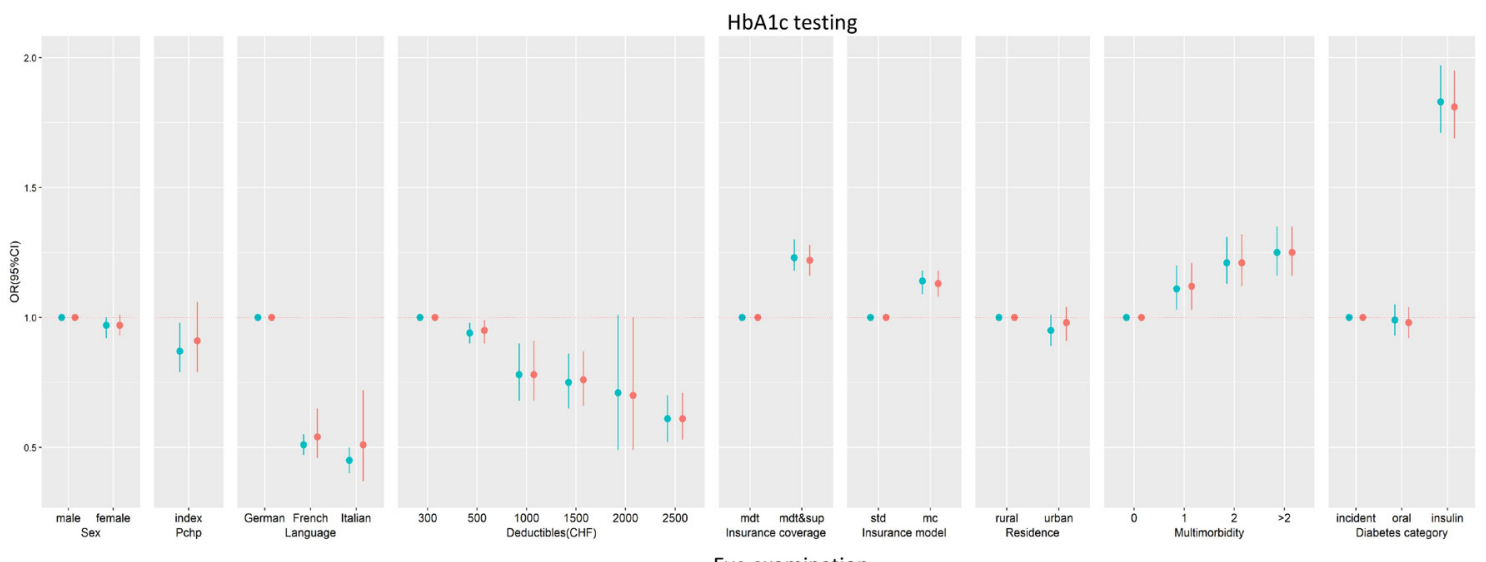

Eye examination

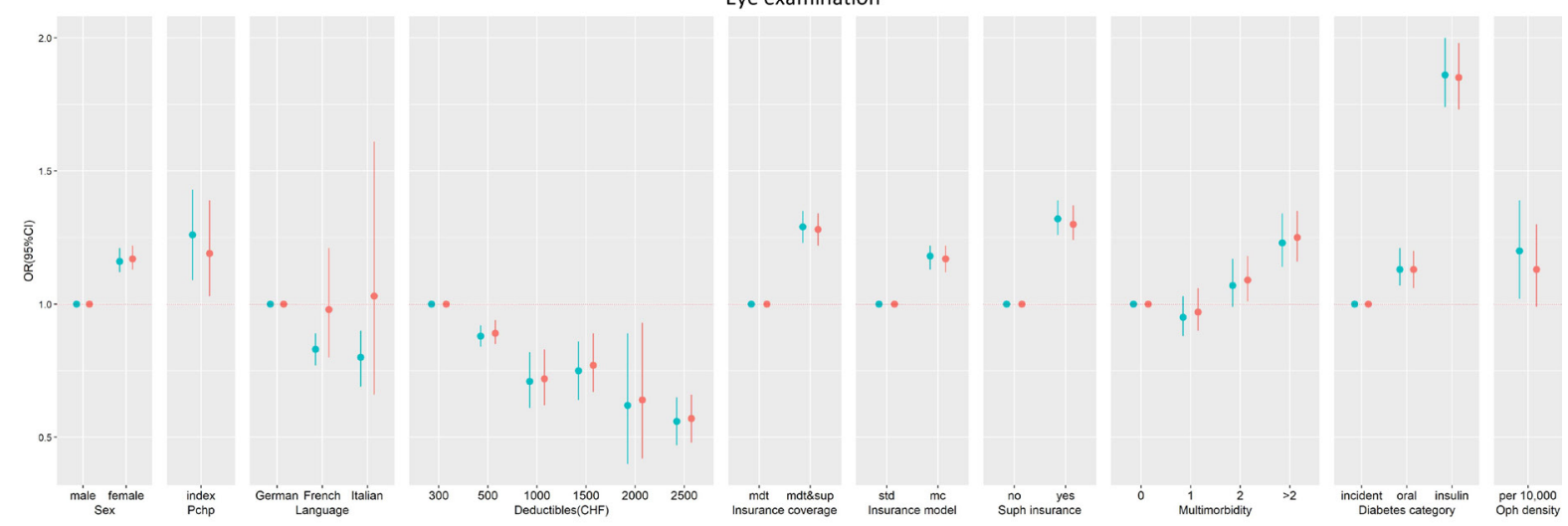

Kidney function examination
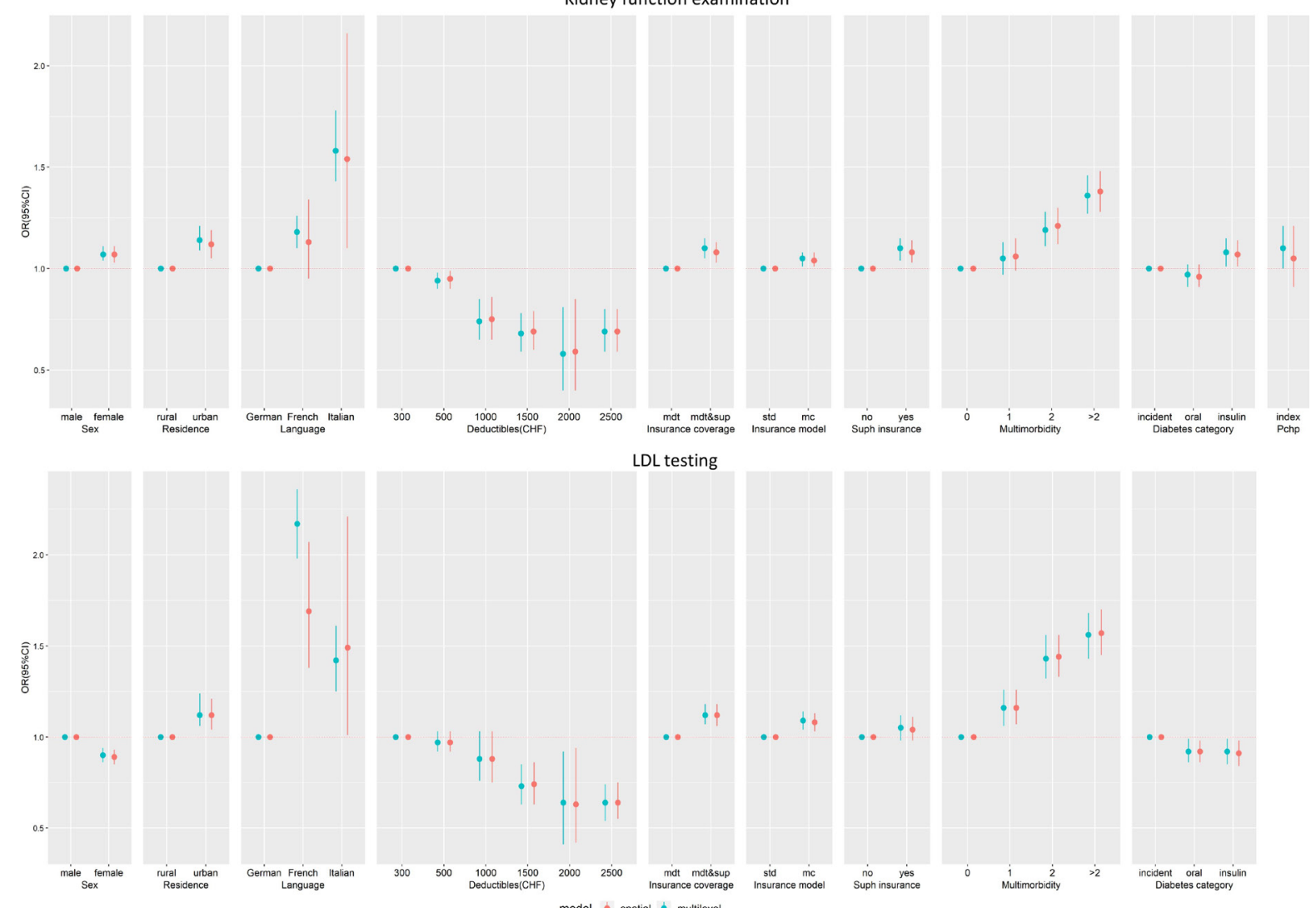

Figure 2 ORs and 95\% Cls of fixed effects in multilevel multivariable models and spatial multilevel models. CHF, Swiss franc; HbA1c, glycated hemoglobin; LDL, low-density lipoprotein; mc, managed care; mdt, mandatory insurance; Oph: ophthalmologist; Pchp, purchasing power; std, standard; sup, supplementary insurance; Suph insurance, supplementary hospital care insurance. Multimorbidity: pharmaceutical cost groups were used to deduce chronic morbidity based on drug use. 

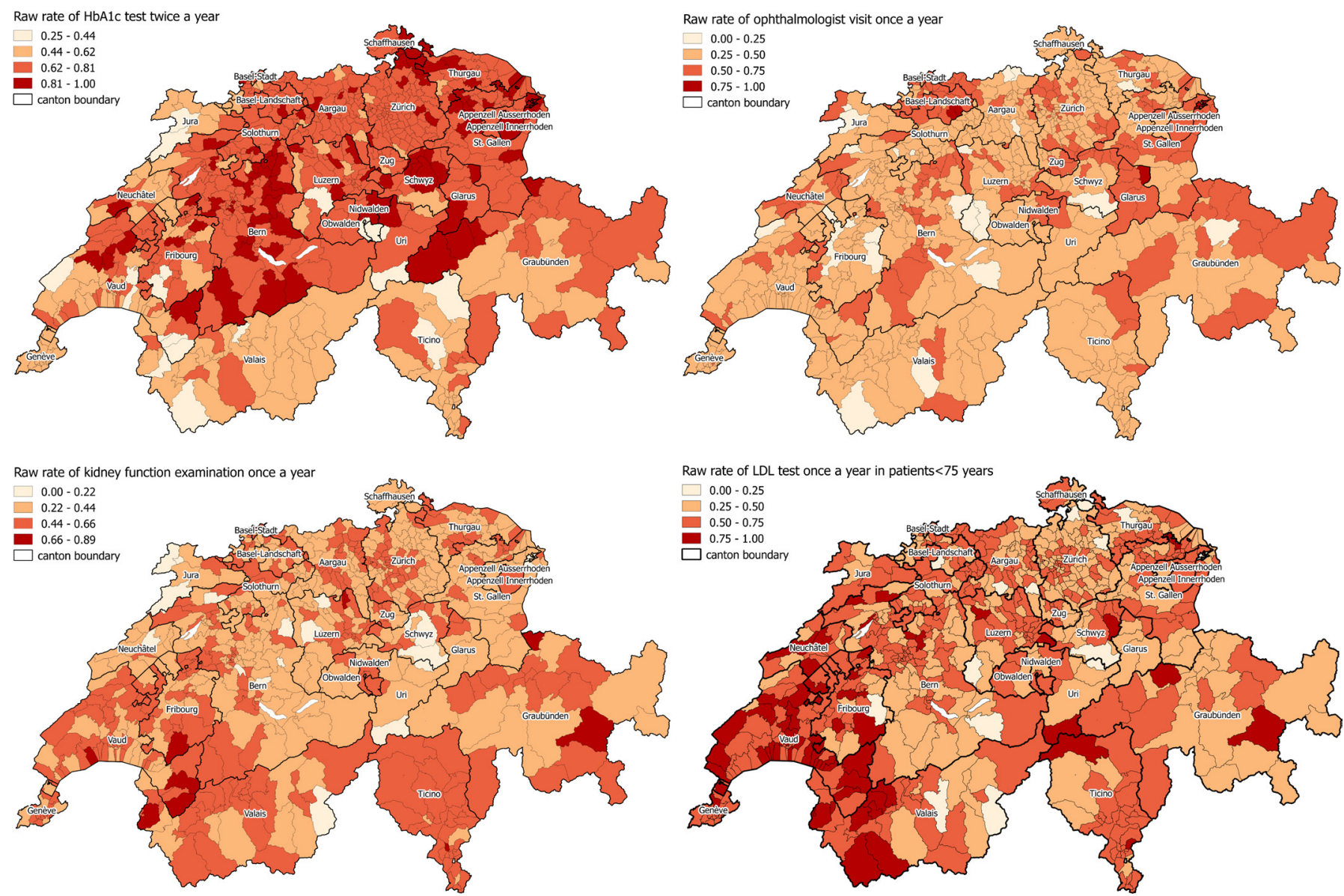

Figure 3 Raw utilization rates of four diabetes management measures across 705 Medstat regions. HbA1c, glycated hemoglobin; LDL, low-density lipoprotein.

to the recommendation in one specific Medstat region compared with the average odds in the whole of Switzerland. We observed spatial clusters of better adherence in the northeast and middle parts of the country for HbAlc testing, in the north and east parts for eye examination, in the southwest and southeast parts for kidney function examination, and in the southwest part for LDL testing.

\section{DISCUSSION}

Observed utilization rates in 2014 in Switzerland of four strongly recommended measures in diabetes management were $69.6 \%$ for biannual HbAlc testing, $44.3 \%$ for annual eye examination, $44.3 \%$ for annual kidney function examination, and $55.5 \%$ for annual LDL testing (in patients below 75 years). Associations between health insurance preferences and utilization were consistent across the four measures. Having supplementary insurance, choosing a lower deductible level, and choosing a managed care insurance model were positively associated with being adherent to the recommendations. After adjusting for all available influencing factors and spatial autocorrelation, the unexplained regional variation was only moderate. There was no common pattern of spatial clustering visible across the four studied measures.
The observed utilization rates suggest that the underlying recommendations were not being followed perfectly. In a previous study using year 2011 to 2013 data from the same data source, similar utilization rates were reported: $70.0 \%$ of patients had biannual HbAlc testing, $44.2 \%$ an annual eye examination, $12 \%$ both serum creatinine and albuminuria testing annually, and $59.0 \%$ an annual lipid profile (total cholesterol, HDL and LDL, and triglycerides). ${ }^{13}$ The much higher rate of annual kidney function examination found in our study was mainly due to the use of a different definition of kidney function examination-a serum creatinine and/ or albuminuria test.

Overall, few studies assessing the utilization of management measures recommended for diabetes patients exist, and some with discrepant findings. Some studies from the USA, Japan and Italy are directly comparable with ours as they reported on the utilization of at least one of our four measures of interest. For HbAlc testing, one study conducted in Texas, USA, reported a $54.8 \%$ biannual utilization rate ${ }^{36}$ while the utilization rate in an Italian study was relatively low (33.9\%) ${ }^{37}$ By contrast, a study from Japan using claims data found an annual utilization rate of $95.8 \%{ }^{38}$ For eye examination, studies in the USA reported utilization rates of $15.3 \%$ (using claims 

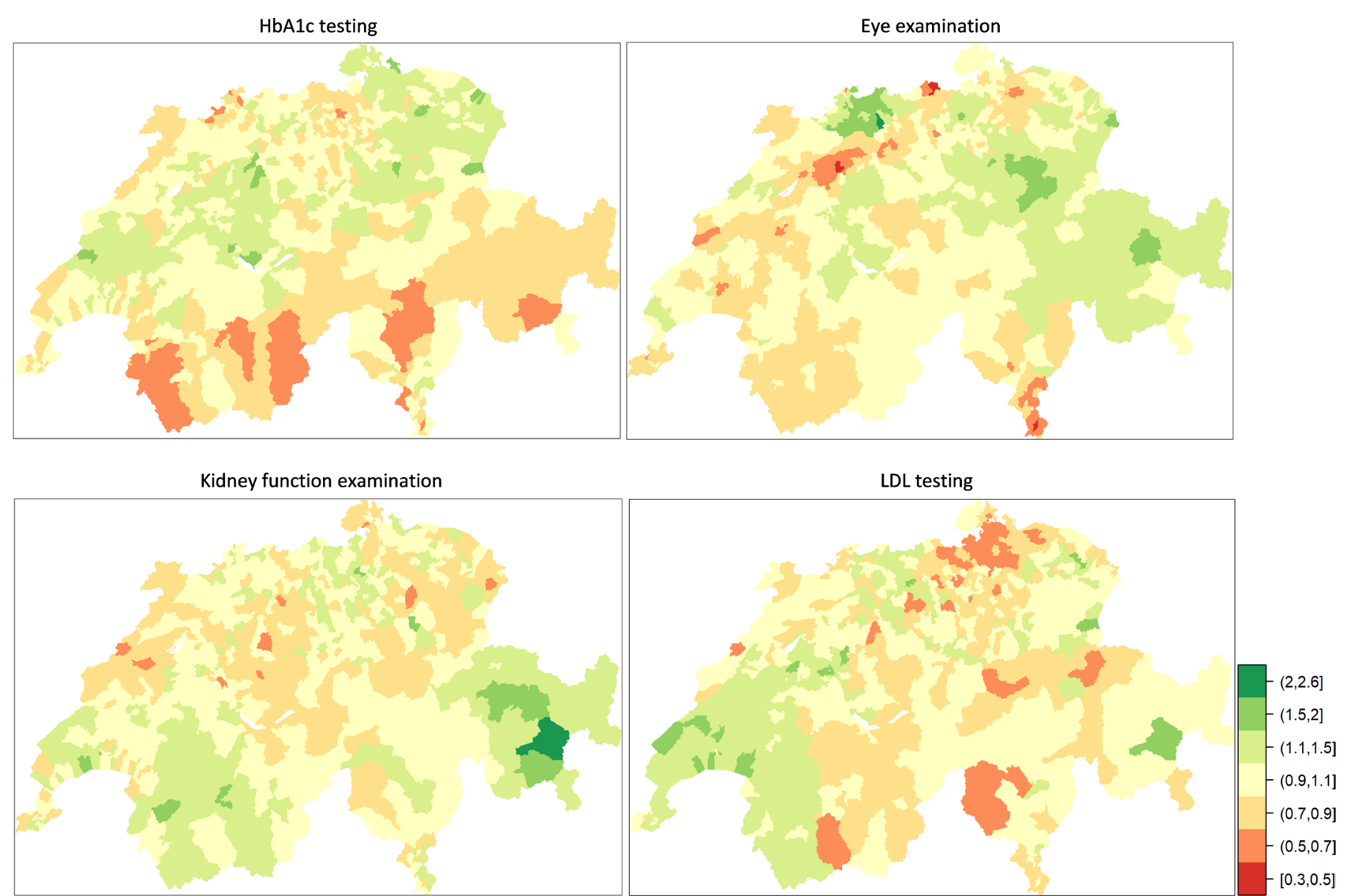

Figure 4 Unexplained variation in the utilization of four diabetes management measures in spatial multilevel models (OR values represent the odds of being adherent to the recommendation in one specific region compared with the average odds in the whole of Switzerland). HbA1c, glycated hemoglobin; LDL, low-density lipoprotein.

data), ${ }^{39} 70 \%$ (using telephone survey data), ${ }^{40}$ and $75 \%$ (data from rural Latinos). ${ }^{41}$ A Japanese study reported a utilization rate of $35.6 \%,{ }^{38}$ and the rate in the Italian study was even lower $(15.6 \%) .{ }^{37}$ The Italian study also reported a utilization rate of LDL testing of $52.1 \%$, which was similar to the finding in our study. ${ }^{37}$ However, these different reports may not be entirely comparable with our study since the data sources and definitions of adherence to recommendations were different.

Patients' sociodemographics were associated with healthcare utilization. The probability of undergoing the four recommended measures was generally high between age 50 and 80 years, and decreased strongly thereafter. This was expected because the elderly may have more barriers to accessing healthcare services due to poor health status. Moreover, the measures may become less important in the elderly as comorbidities and life expectancy affect priority setting and the benefit of preventing long-term complications. Women were more likely to undergo eye examination and kidney function examination in our study, which was consistent with previous findings. ${ }^{38} 4243$ However, women were less likely to undergo annual LDL testing, which might be due to more attention to the risk of cardiovascular disease in men. Myocardial infarction and related conditions have traditionally been perceived as predominantly male diseases. Living in an urban area was positively associated with more utilization of annual kidney function examination and LDL testing, which may be partly explained by easier access to healthcare facilities than in rural areas. The language region effects on the utilization of the four measures found in the present study indicated that the language region plays an important role in influencing healthcare utilization, which might be due to different culture and norms in each language region. ${ }^{44}$

One of the key findings in the present study was the association between health insurance preferences and utilization of diabetes management measures, in a setting with mandatory insurance and universal access to care. Very few studies have explored the effect of health insurance-related factors on services utilization in patients with diabetes. Most of the available studies only concluded that uninsured patients were less likely to use healthcare services than insured patients or patients with private insurance. ${ }^{3640}$ While non-insurance does practically not occur in Switzerland, foregoing healthcare due to out-ofpocket payments is a well-documented phenomenon. ${ }^{46}$ This is one of the first studies to look into potential influences of health insurance characteristics on utilization of measures on diabetes management in detail. Overall, we 
found consistent effects of health insurance characteristics on utilization across the four measures of interest, and they persisted after controlling for other important influences such as age, health status, and to some extent income (defined by regional purchasing power index). Patients with higher deductibles tend to be healthier and willing to take more risks, and some invoices may be missed in these patients, which may partially explain our observation of lower utilization of the measures of interest. However, higher out-of-pocket costs may also make patients more reluctant to use these measures, which would make high deductibles a financial barrier to recommended healthcare ${ }^{47}$ Similarly, patients having supplementary insurance may be wealthier, and on average more health conscious. Thus, they may tend to seek care more frequently and regularly, as observed in our study. Patients choosing a managed care model had more utilization of the measures studied than patients choosing an insurance model offering completely free physician choice. This finding is of great interest because it may indicate that strengthening a coordinative role of primary care physicians in managed care and providing financial incentives to the insured for choosing such models may also positively impact certain healthcare utilization indicators or outcomes. More health insurance incentives for participation in managed care models could be considered to achieve optimized healthcare utilization.

Presence of comorbidities was associated with more utilization, which may be due to more health awareness and regular contact with healthcare providers. The finding of the lower uptake of LDL testing among prevalent cases was unexpected, as we would have expected prevalent patients to be more adherent to disease management and treatment compared with new patients. ${ }^{48}$ The ophthalmologist density covariate reflected the access to eye examination services, and thus partly explained the higher utilization of eye examinations in patients living in regions with more ophthalmologists.

The unexplained geographic variation of utilization across small regions after adjusting for all available factors was only moderate for all four measures. One possible reason could be that we were unable to control for locally specific factors in our models. For example, physicianlevel factors such as age, years in practice, and the awareness of and attitude toward clinical guidelines and recommendations vary across physicians and could affect the communication with patients and finally the patients' behaviors. ${ }^{49}$ In addition, some patient-level characteristics were not captured in our data source, for example, educational level or marital status, as well as patients' preferences, which were demonstrated to be potentially related to the utilization of healthcare services. ${ }^{40}$ By mapping out the unexplained spatial variation, we noted that the spatial patterns of regional variation were inconsistent across the four measures studied. These patterns indicated that the utilization of the four measures strongly recommended to patients with diabetes differ substantially within Switzerland. The spatial variation of utilization might be even less prominent after controlling for more potentially influential factors unmeasured in the present study, such as physician characteristics which could not be captured from the claims data. Combination of different data sources may serve as a promising approach in future studies.

In addition to the limitations mentioned above, further potential weaknesses should be noted about this study. First, the health insurance claims data have limited clinical information; for example, outpatient diagnoses are lacking. The study population was selected according to the prescription of any diabetes medication, which may have led to some misclassification of prevalent and incident cases; this might partially account for the unexpected finding of more utilization of LDL testing in incident patients. It was impossible to distinguish between type 1 and type 2 diabetes. Patients with type 1 diabetes are a small fraction (approximately $8 \%$ in Switzerland in $2014^{50}$ ); they normally get the illness when they are young, tend to be well treated by specialists, and are generally better at self-management. Due to the high costs of insulin injections and the associated medical supplies and devices, choosing a low deductible level is expected in patients with type 1 diabetes. Such different behaviors may have had an impact on utilization of the four measures and influenced our results to a certain degree. Besides, the laboratory test results were not available from claims data and it was impossible to estimate the proportion of targets achieved for the diabetes management measures. Second, we used claims data from a single health insurer. Enrollees of other health insurers might theoretically have different characteristics and show different healthcare use patterns. However, the results presented were based on a population of 1.2 million covering all regions in Switzerland. The benefit package of the mandatory health insurance is defined at the federal level and is the same for all health insurers. Thus, we expect little deviation of enrollees' features compared with the total Swiss population, and the results should be generalizable to the whole of Switzerland.

In conclusion, we observed that the utilization of four diabetes management measures was not optimal in Switzerland although these measures have been recommended broadly and are based on strong evidence. Sociodemographics, health insurance preferences, and clinical characteristics were associated with their utilization. The presence of supplementary insurance, a lower deductible level, and participation in a managed care plan were associated with higher utilization, consistently across the four measures. After controlling for available factors and spatial autocorrelation, maps of remaining variation indicated inconsistent patterns of utilization in the four measures. Our findings indicate that the uptake of strongly recommended measures for diabetes management could possibly be optimized by providing further incentives to insured and care providers through insurance scheme design. By contrast, due to the absence of 
marked regional variation patterns, we conclude that there may be only limited potential for improvement by targeted regional intervention (eg, awareness and promotion campaigns). Moreover, our novel approach aids in the identification of geographic variation and influencing factors of healthcare services use in Switzerland and comparable settings worldwide.

\section{Author affiliations}

${ }^{1}$ Department of Epidemiology, Epidemiology, Biostatistics and Prevention Institute, University of Zurich, Zurich, Switzerland

${ }^{2}$ Department of Geography, University of Zurich, Zurich, Switzerland

${ }^{3}$ Division of Occupational and Environmental Medicine, Department of

Epidemiology, Epidemiology, Biostatistics and Prevention Institute, University of

Zurich and University Hospital Zurich, Zurich, Switzerland

${ }^{4}$ Department of Health Sciences, Helsana Versicherungen AG, Dübendorf, Switzerland

${ }^{5}$ Division of General Practice, University Medical Center Freiburg, Freiburg, BadenWürttemberg, Germany

${ }^{6}$ Departments of Biostatistics and Epidemiology, Epidemiology, Biostatistics and Prevention Institute, University of Zurich, Zurich, Switzerland

Contributors MS, VvW, and HD developed the underlying study program and generated the idea of the present study. $\mathrm{BB}, \mathrm{EB}, \mathrm{CB}$, and $\mathrm{AU}$ did data preparation and data management. WW, $\mathrm{OG}$, and JB performed statistical analysis and wrote the main manuscript text. All authors together defined the analysis methodology, interpreted the statistical results, and critically reviewed the manuscript.

Funding The study was supported by the Swiss National Science Foundation (SNSF) National Research Program "Smarter Health Care" (NRP 74), as part of project number 26, grant number 407440_167349.

Map disclaimer The depiction of boundaries on the maps in this article do not imply the expression of any opinion whatsoever on the part of BMJ (or any member of its group) concerning the legal status of any country, territory, jurisdiction or area or of its authorities. The maps are provided without any warranty of any kind, either express or implied.

\section{Competing interests None declared.}

Patient consent for publication Not required.

Provenance and peer review Not commissioned; externally peer reviewed.

Data availability statement Data may be obtained from a third party and are not publicly available. The data underlying this study cannot be shared publicly because they are the property of Helsana (https://www.helsana.ch/en/helsanagroup), and have restricted public access on grounds of patient privacy. The data are managed by Helsana and subsets of the database are available for researchers after request and under specific conditions. Data are available from Helsana ( gesundheitskompetenz@helsana.ch) for researchers who meet the criteria for access to confidential data. Helsana will consider the possibilities of the research proposal and decide to grant access if the research questions can be answered with use of the Helsana data. When requests are granted, data are accessible only in a secure environment.

Open access This is an open access article distributed in accordance with the Creative Commons Attribution Non Commercial (CC BY-NC 4.0) license, which permits others to distribute, remix, adapt, build upon this work non-commercially, and license their derivative works on different terms, provided the original work is properly cited, appropriate credit is given, any changes made indicated, and the use is non-commercial. See: http://creativecommons.org/licenses/by-nc/4.0/.

ORCID iDs

Wenjia Wei http://orcid.org/0000-0002-1977-8416

Beat Brüngger http://orcid.org/0000-0001-6173-5375

\section{REFERENCES}

1 WHO. Global report on diabetes, 2016. Available: https://www.who. int/diabetes/global-report/en/

2 Diabetes a 'defining issue for global public health', 2016. Available: https://www.swissinfo.ch/eng/world-health-study_diabetes-adefining-issue-for-global-public-health/42070902
3 Tamayo T, Rosenbauer J, Wild SH, et al. Diabetes in Europe: an update. Diabetes Res Clin Pract 2014;103:206-17.

4 Kriterien für "gutes" Disease Management Diabetes in der Grundversorgung. Schweizerische Gesellschaft für Endokrinologie und Diabetologie., 2017. Available: https://www.sgedssed.ch/ diabetologie/praxis-tools-fuer-die-grundversorgung?key $=1-8 \&$ cHash $=c 5691$ c4b4cceOfaa42491499f3f2e5ab

5 American Diabetes Association. Standards of Medical Care in Diabetes -2019 Abridged for Primary Care Providers. Clin Diabetes 2019;37:11-34.

6 Rydén L, Grant PJ, Anker SD, et al. Esc guidelines on diabetes, prediabetes, and cardiovascular diseases developed in collaboration with the EASD: the task force on diabetes, pre-diabetes, and cardiovascular diseases of the European Society of cardiology (ESC) and developed in collaboration with the European association for the study of diabetes (EASD). Eur Heart J 2013;34:3035-87.

7 Ahmann AJ. Guidelines and performance measures for diabetes. Am J Manag Care 2007;13:S41-6.

8 De Berardis G, Pellegrini F, Franciosi M, et al. Quality of care and outcomes in type 2 diabetic patients: a comparison between general practice and diabetes clinics. Diabetes Care 2004;27:398-406.

9 Fleming BB, Greenfield S, Engelgau MM, et al. The Diabetes Quality Improvement Project: moving science into health policy to gain an edge on the diabetes epidemic. Diabetes Care 2001;24:1815-20.

10 Giorda C, Picariello R, Nada E, et al. The impact of adherence to screening guidelines and of diabetes clinics referral on morbidity and mortality in diabetes. PLoS One 2012;7:e33839.

11 Giorda CB, Picariello R, Nada E, et al. Comparison of direct costs of type 2 diabetes care: different care models with different outcomes. Nutr Metab Cardiovasc Dis 2014;24:717-24.

12 Oh S-W, Lee HJ, Chin HJ, et al. Adherence to clinical practice guidelines and outcomes in diabetic patients. Int $J$ Qual Health Care 2011;23:413-9.

13 Huber CA, Brändle M, Rapold R, et al. A set of four simple performance measures reflecting adherence to guidelines predicts hospitalization: a claims-based cohort study of patients with diabetes. Patient Prefer Adherence 2016;10:223-31.

14 Appleby JRV, Frosini F, Bevan G, et al. Variations in health care: the good, the bad and the inexplicable. King's Fund 2011.

15 Wennberg JE. Tracking medicine. 9. Oxford: Oxford University Press, 2010.

16 Wennberg JE. Unwarranted variations in healthcare delivery: implications for academic medical centres. BMJ 2002;325:961-4

17 Blozik E, Signorell A, Reich O. How does hospitalization affect continuity of drug therapy: an exploratory study. Ther Clin Risk Manag 2016;12:1277-83.

18 Reich O, Rapold R, Thöni M, Flatscher-Thoni M. An empirical investigation of the efficiency effects of integrated care models in Switzerland. Int J Integr Care 2012;12:e2.

19 Ulyte A, Bähler C, Schwenkglenks M, et al. Measuring diabetes guideline adherence with claims data: systematic construction of indicators and related challenges. BMJ Open 2019;9:e027138.

20 Huber CA, Szucs TD, Rapold R, et al. Identifying patients with chronic conditions using pharmacy data in Switzerland: an updated mapping approach to the classification of medications. BMC Public Health 2013;13:1030.

21 Medstat-Regionen. Bundesamt für Statistik, 2008. Available: https://www.bfs.admin.ch/bfs/de/home/statistiken/gesundheit/ nomenklaturen/medsreg.html

22 Srinivasan S. Local and global spatial statistics. encyclopedia of GIS. Boston, MA: Springer US, 2008: 615-15.

23 Best NG, Best NG, Carlin BP, et al. Bayesian measures of model complexity and fit. J Royal Statistical Soc B 2002;64:583-639.

24 Larsen K, Merlo J. Appropriate assessment of neighborhood effects on individual health: integrating random and fixed effects in multilevel logistic regression. Am J Epidemiol 2005;161:81-8.

25 Larsen K, Petersen JH, Budtz-Jørgensen E, et al. Interpreting parameters in the logistic regression model with random effects. Biometrics 2000;56:909-14.

26 Ohlsson $\mathrm{H}$, Lindblad $\mathrm{U}$, Lithman $\mathrm{T}$, et al. Understanding adherence to official guidelines on statin prescribing in primary health care-a multi-level methodological approach. Eur J Clin Pharmacol 2005:61:657-65.

27 Panczak R, Held L, Moser A, et al. Finding big shots: small-area mapping and spatial modelling of obesity among Swiss male conscripts. BMC Obesity 2016;3:10.

28 Panczak R, Moser A, Held L, et al. A tall order: small area mapping and modelling of adult height among Swiss male conscripts. Economics \& Human Biology 2017;26:61-9.

29 Riesen M, Konstantinoudis G, Lang $\mathrm{P}$, et al. Exploring variation in human papillomavirus vaccination uptake in Switzerland: a multilevel 
spatial analysis of a national vaccination coverage survey. BMJ Open 2018;8:e021006.

30 Schrödle B, Held L. A primer on disease mapping and ecological regression using INLA. Comput Stat 2011;26:241-58.

31 Rue H, Martino S, Chopin N. Approximate Bayesian inference for latent Gaussian models by using integrated nested Laplace approximations. J Royal Stat Soc Series B 2009;71:319-92.

32 R Core Team. R: a language and environment for statistical computing. Vienna, Austria: R Foundation for Statistical Computing, 2018. https://www.r-project.org/

33 Charlton C, Rasbash J, Browne WJ, et al. MLwiN version 3.042019.

34 QGIS Development Team. QGIS Geographic Information System. Open Source Geospatial Foundation Project. Available: https://qgis. org/en/site/ [Accessed 18 Jun 2019].

35 Anselin L, Syabri I, Kho Y. GeoDa: an introduction to spatial data analysis. Geogr Anal 2006;38:5-22.

36 Nepal V, Banerjee D. A1c testing and its sociodemographic predictors: implications for diabetes self-management programs. Health Serv Res Manag Epidemiol 2014;1:2333392814547129.

37 Corrao G, Rea F, Di Martino M, et al. Effectiveness of adherence to recommended clinical examinations of diabetic patients in preventing diabetes-related hospitalizations. Int J Qual Health Care 2019;31:464-72.

38 Tanaka H, Tomio J, Sugiyama T, et al. Process quality of diabetes care under favorable access to healthcare: a 2-year longitudinal study using claims data in Japan. BMJ Open Diab Res Care 2016;4:e000291.

39 Benoit SR, Swenor B, Geiss LS, et al. Eye care utilization among insured people with diabetes in the U.S., 2010-2014. Diabetes Care 2019;42:427-33.

40 Nelson KM, Chapko MK, Reiber G, et al. The association between health insurance coverage and diabetes care; data from the 2000 Behavioral Risk Factor Surveillance System. Health Serv Res 2005;40:361-72.
41 Sadowski D, Devlin M, Hussain A. Better care at safety net providers? Utilization of recommended standards of diabetes care for rural Latinos in one Midwestern state. $J$ Health Care Poor Underserved 2011;22:995-1013.

42 Shalev V, Chodick G, Heymann AD, et al. Gender differences in healthcare utilization and medical indicators among patients with diabetes. Public Health 2005;119:45-9.

43 Krämer HU, Rüter G, Schöttker B, et al. Gender differences in healthcare utilization of patients with diabetes. Am J Manag Care 2012;18:362-9.

44 Delco F, Michetti P, Beglinger C, et al. Health care resource utilization and costs of NSAID-induced gastrointestinal toxicity. A populationbased study in Switzerland. Digestion 2004;69:10-19.

45 Panczak R, Luta X, Maessen M, et al. Regional variation of cost of care in the last 12 months of life in Switzerland: smallarea analysis using insurance claims data. Medical care 2017;55:155-63.

46 Wolff H, Gaspoz JM, Guessous I. Health care renunciation for economic reasons in Switzerland. Swiss Med Wkly 2011;141:w13165.

47 Guessous I, Gaspoz JM, Theler JM, et al. High prevalence of forgoing healthcare for economic reasons in Switzerland: a population-based study in a region with universal health insurance coverage. Prev Med 2012;55:521-7.

48 Torre C, Guerreiro J, Longo P, et al. Effect of different methods for estimating persistence and adherence to new glucose-lowering drugs: results of an observational, inception cohort study in Portugal. Patient Prefer Adherence 2018;12:1471-82.

49 Mehta S, Mocarski M, Wisniewski T, et al. Primary care physicians' utilization of type 2 diabetes screening guidelines and referrals to behavioral interventions: a survey-linked retrospective study. BMJ Open Diab Res Care 2017;5:e000406.

50 Swiss Diabetes Association. Facts + Figures, 2014. Available: http:// www.diabetesschweiz.ch/diabetes/facts-figures/ 